Publ. RIMS, Kyoto Univ. Ser. A

Vol. 3 (1967), pp. 151-166

\title{
An algebra of pseudo dinference schemes and its application
}

\author{
By \\ Masaya YAmaguti* and Tatsuo NoGi**
}

\section{Introduction}

In this paper we discuss an algebra of one-parameter families of bounded operators mapping the space $L^{2}$ of square integrable vector valued functions into itself. As we know, the algebra of pseudo differential operators by Caldéron-Zygmund and its extensions are very useful devices to obtain the energy inequality for the Cauchy problem of nonsymmetric hyperbolic systems of differential equations [1] [2] [4] [6] [7]. Analogously we introduce the algebra of one-parameter families of bounded operators for the purpose of getting some local energy inequality to assure the stability of a finite difference scheme for regularly nonsymmetric hyperbolic systems [3]. The authors are greatly indebted to the advice of Prof. S. Matsuura particularly for the formalism in section 3. We wish to thank him for this advice.

\section{An example}

In the theory of pseudo differential operators, we have a special operator denoted by $\Lambda$ which is the Fourier transform of the multiplication operator $|\xi|$ in $L_{\xi}^{2}$ where $\xi$ means a real vector $\left(\xi_{1}, \cdots, \xi_{n}\right)$ in $R_{\xi}^{n}$. This operator plays an important role in the theory of differential equations with variable coefficients. In that case the commutator $a(x) \Lambda-\Lambda a(x)$, where $a(x)$ is a smooth bounded function, is a bounded operator in the $L^{2}$ sense. While in the $L^{2}$ theory of finite difference

Received May 27, 1967.

Communicated by S. Matsuura.

* Department of Applied Mathematics and Physics, Kyoto University.

** Same as above. 
schemes with variable ccefficients, a commutatcr $a(x) T-T a(x)$, where $T$ is a translation operator defined by $T u(x)=u(x+h e)$ ( $h$ is a positive number, $e$ is some unit $n$-vector), have the property that it is a one-parameter family of bounded operators with norm $O(h)$. This property corresponds to the boundedress of operators in the theory of pseudo differential operators. Now we ask what is a family corresponding to the operator $\Lambda$. Maybe there are several families corresponding to this operator. Here we consider one example which is defined in the following way:

$$
\Lambda_{h}=\mathscr{E}^{-1}|\sin h \xi| \mathscr{F}
$$

where $\mathscr{I}$ mears usual Fcurier transformation and $\sin h \xi$ means a vector $\left(\sin h \xi_{1}, \sin h \xi_{2}, \cdots, \sin h \xi_{n}\right) .|\sin h \xi|$ means, of course, the absolute value of this vector.

We shall show first this family of operators has a similar property to that of $T$. We assume that $a(x)$ is smooth and is equal to a constant for large $|x|$. Then we can prove that $a(x) \Lambda_{h}-\Lambda_{k} a(x)$ is a family of bounded operators with norm $O(h)$. Because, for every square integrable function $u$ putting $a_{1}(x)=a(x)-a(\infty)$ ), we get

$$
\begin{aligned}
& \begin{aligned}
\mathscr{F}[a(x) & \left.\Lambda_{h}-\Lambda_{h} a(x)\right] u \\
& =\mathscr{F}\left[a_{1}(x) \Lambda_{h}-\Lambda_{h} a_{1}(x)\right] u \\
& =\int \hat{a}_{1}(\xi-\eta)|\sin h \eta| \hat{u}(\eta) d \eta-\int \hat{a}_{1}(\xi-\eta)|\sin h \xi| \hat{u}(\eta) d \eta \\
& =\int \hat{a}_{1}(\xi-\eta)[|\sin h \eta|-|\sin h \xi|] \hat{u}(\eta) d \eta . \\
\|\left[a_{1}(x)\right. & \left.\Lambda_{h}-\Lambda_{h} a_{1}(x)\right] u\|\leqq\| \int\left|\hat{a}_{1}(\xi-\eta)\right||\sin h \eta-\sin h \xi||\hat{u}(\eta)| d \eta \| \\
= & \int\left|\hat{a}_{1}(\xi-\eta)\right|\left|2 \sin h \frac{(\xi-\eta)}{2}\right|\left|\cos \frac{h(\xi+\eta)}{2}\right||\hat{u}(\eta)| d \eta \|_{\mid} \\
& \leqq h\left|\int_{\mid}\right| \hat{a}_{1}(\xi-\eta)|| \xi-\eta|| \hat{u}(\eta)|d \eta| \\
& \leqq h\left\|\left|\hat{a}_{1}(\xi)\right| \cdot|\xi|\right\| L_{1} \mid\|u\| .
\end{aligned}
\end{aligned}
$$

3. An algebra of one-parameter families of operators

Definition. $A$ one parameter family $H_{h}$ of bounded operators 
mapping $L^{2}$ into itself is called a "null scheme" if it satisfies the following inequality. (And we denote the set of all null schemes by $\left.\Re_{0}\right)$.

$$
\left\|H_{h}\right\|=O(h) .
$$

Next we consider the set $\mathcal{K}$ of $p \times p$ matrix valued functions $k$ defined for $x$ in $R_{x}^{n}$ and $\xi$ in $R_{\xi}^{n}$ with the following properties

i) $k$ is homogeneous of degree zero in $\xi$.

ii) each $k$ is independent of $x$ for $|x|>R ; R$ is a fixed positive constant.

iii) $k(x, \xi)$ belongs to $C^{\infty}\left(R_{x}^{n} \times\left(R_{\xi}^{n}-\{0\}\right)\right)$.

The set $\mathcal{K}$ forms an algebra of matrix valued functions by pointwise addition and multiplication.

Now we associate a one-parameter family of operators $K_{h}$ with each function $k \in \mathcal{K}$ by the following formula:

$$
\mathscr{L}_{h} u=1 . \mathrm{i} . \mathrm{m} . \int e^{2<x, \xi>} k(x, \lambda(h \xi)) \hat{u}(\xi) d \xi \quad u \in L_{\imath}^{2}
$$

where $\lambda(\xi)=\left(\lambda_{1}(\xi), \lambda_{2}(\xi), \cdots, \lambda_{n}(\xi)\right)$ is a real valued vector function which satisfies the following two conditions:

$$
\begin{aligned}
& |\lambda(\xi)-\lambda(\eta)| \leqq C|\xi-\eta|,|\lambda(\xi)| \leqq M \text { for } \xi, \eta \in R_{\xi}^{n} \\
& \lim _{h \rightarrow \infty} \frac{\lambda(h \xi)}{h}=\xi \text { for } \xi \in R_{\xi}^{n} .
\end{aligned}
$$

The existence of the limit in the mean in (3) is based on the following estimate (4) and the expansion in lemma 2 below (see (10)).

$$
\left\|K_{h}\right\| \leqq \sup _{\substack{x \in R^{n} \\ \xi \in \omega \\|\beta| \leq m}}\left|D_{\xi}^{\beta} k(x, \xi)\right| .
$$

with $\omega$ a fixed compact in $R_{\xi}^{n}-\{0\}, m$ an integer depending only on the dimension $n$ of the space, $C$ a constant.

Now we consider the algebra of one-parameter families of bounded operators mapping $L^{2}$ into itself generated by $K_{h}$ defined above. We denote this algebra by $\mathscr{L}$ and we call it the algebra of "pseudo difference schemes." 
Next we need to define an operator $\Lambda_{k}$ (of which one example was stated in section 2) by the following:

$$
\Lambda_{h}=\mathscr{F}^{-1}|\lambda(h \xi)| \mathscr{F} \text {. }
$$

Definition. A family $K_{h}$ which belongs to $\mathscr{P}$ is called "negligible" if $\Lambda_{h} K_{h}$ and $K_{h} \Lambda_{h}$ belong to $\Omega_{0}$. The set of all negligible schemes is denoted by $\mathcal{R}$.

Lemma 1. If $a(x)$ belongs to $\mathcal{K}$, then $\left[a(x), \Lambda_{k}\right]=a(x) \Lambda_{k}$ $-\Lambda_{h} a(x)$ is a null scheme.

Proof is completely similar to the proof for the case $\lambda_{h}(x)=\sin h \xi$ which is stated in section 2. We only used the condition $(\alpha)$ of $\lambda_{h}(\xi)$. In fact, for all $u \in L_{x}^{2}$, there exists a constant $C$

$$
\left\|\left[a(x), \Lambda_{h}\right] u\right\|_{i} \leqq C\left\|\hat{a}_{1}(\xi)|\xi|\right\|_{L^{1}} \cdot\|u\|_{L^{2}} \cdot h, \quad a_{1}(x)=a(x)-a(\infty) .
$$

Lemma 2. (P. D. Lax)

Every $k$ in $\mathcal{K}$ can be expanded in a series

$$
k(x, \xi)=\sum_{\alpha} a_{\alpha} \exp \left(i\left\langle\alpha, \frac{\xi}{|\xi|}\right\rangle\right)
$$

a varying over all multiindices so that the series, as well as the differentiated series with respect to $x$ or $\xi$, converge uniformly. For the proof see [4]. Therefore we see that the following finite sums of special kernels are dense in $\mathcal{K}$ with respect to the topology of $C^{1}\left(R_{x}^{n} \times\left(R_{\xi}^{n}-\{0\}\right)\right)$ :

$$
\sum_{\alpha}^{\text {finite }} a_{\alpha}(x) k_{\alpha}(\xi), \quad k_{\alpha}(\xi), a_{\alpha}(x) \in \mathcal{K} .
$$

Here we can assume that $k_{\alpha}(\xi)$ are scalar ones according to the lemma 2.

We try to compute the corresponding families $A_{\alpha}$ and $K_{k \alpha}$ in $\mathscr{P}$ for the kernels $a_{\alpha}(x)$ and $k_{\alpha}(\xi)$, then we get

$$
\begin{aligned}
& A_{\alpha} u=a_{\alpha}(x) u, \quad u \in L_{x}^{2} \\
& K_{k \alpha} u=\mathscr{F}^{-1} k_{\alpha}(\lambda(h \xi)) \mathscr{F} u, \quad u \in L_{x}^{2} .
\end{aligned}
$$

Consequently we can express the family $K_{h}$ associated with general element $k(x, \xi)$ in $\mathcal{K}$ in the following manner: 


$$
K_{h}=\sum_{\infty} A_{\alpha} K_{h \alpha}
$$

because of the continuity of mapping $\mathscr{M}: \mathcal{K} \rightarrow \mathscr{L}$ with respect to $C^{m}$ topology (see (4)).

Now we state a lemma:

Lemma 3. If $K_{h}$ is a family in $\mathcal{Q}$ assocaited with $k$ in $\mathcal{K}$, then the commutator $\left[K_{h}, \Lambda_{h}\right]$ is a null scheme.

Proof. First we remark that if $\left\|\left[K_{h}^{(n)}, \Lambda_{h}\right]\right\| \leqq C h$ with $C$ independent of $n$, and if $K_{h}^{(n)} \rightarrow K_{h}(n \rightarrow+\infty)$ with respect to the operator norm, then $\left[K_{k}, \Lambda_{k}\right]$ belongs to $\mathscr{M}_{0}$. Therefore it suffices to prove this lemma for the case of $K_{l}^{(n)}$ associated with the finite sum:

$$
k^{(n)}(x, \xi)=\sum_{\alpha}^{f_{\text {nnt }} \text { te }} a_{\alpha}(x) k_{\alpha}(\xi) .
$$

We have already

$$
\left\|a_{\alpha}(x) \Lambda_{h}-\Lambda_{h} a_{\alpha}(x)\right\| \leqq h\left\|\hat{a}_{1 \alpha}(\xi)|\xi|\right\|_{L_{1}}
$$

but we also have

$$
\left\|\hat{a}_{1 a}(\xi)|\xi|\right\|_{L_{1}} \leqq \frac{M}{|\alpha|^{k}} \text { for some constant } M
$$

because using the fact that the support of $a_{1 \alpha}(x)$ are contained in a fixed compact

$$
\begin{aligned}
\left\|\hat{a}_{1 \alpha}(\xi)|\xi|\right\|_{L_{1}} & \leqq\left(\int \frac{d \xi}{(1+|\xi|)^{2 n}} \cdot \int(1+|\xi|)^{2(n+1)}\left|\hat{a}_{1 \alpha}(\xi)\right|^{2} d \xi\right)^{1 / 2} \\
& \leqq M \sup _{\substack{x \\
|\beta| \leqq n+1}}\left|D_{\alpha}^{\beta} a_{1 \alpha}(x)\right| \\
& \leqq \frac{M}{(1+|\alpha|)^{k}} .
\end{aligned}
$$

Here we can take $k$ large enough since $a_{1 \alpha}(x) \in C_{0}^{\infty}$. Then we get

$$
\left\|\left[K_{h}^{(n)}, \Lambda_{h}\right]\right\| \subseteq C h
$$

with $C$ independent of $n$.

Now we state another important lemma

Lemma 4. If $a(x)$ and $k(\xi)$ are in $\mathcal{K}$, then the commutator $\left[A, K_{h}\right]$ of $A$ and $K_{h}$ is negligible. 
Proof. For every $u \in L_{x}^{2}$, we have

$$
\begin{aligned}
& \left\|\Lambda_{h}\left[A, K_{h}\right] u\right\|=\left\|\left(\Lambda_{h} A K_{h}-\Lambda_{h} K_{h} A\right) u\right\| \\
& =\left\|\int \hat{a}(\xi-\eta)|\lambda(h \xi)|[k(\lambda(h \eta))-k(\lambda(h \xi))] \hat{u}(\eta) d \eta\right\| \\
& \leqq\|\hat{a}(\xi-\eta)[|\lambda(h \xi)|-|\lambda(h \eta)|] k(\lambda(h \eta)) \hat{u}(\eta) d \eta\| \\
& +\left\|\int_{\|} \hat{a}(\xi-\eta)[|\lambda(h \eta)| k(\lambda(h \eta))-|\lambda(h \xi)| k(\lambda(h \xi))] \hat{u}(\eta) d \eta\right\| \\
& \leqq \int_{\mid}|\hat{a}(\xi-\eta)||\lambda(h \xi)-\lambda(h \eta)||k(\lambda(h \eta))||\hat{u}(\eta)| d \eta \| \\
& +\int_{11}^{\|}|\hat{a}(\xi-\eta)||\lambda(h \xi)-\lambda(h \eta)| \times \\
& \times\left|\frac{|\lambda(h \eta)| k(\lambda(h \eta))-|\lambda(h \xi)| k(\lambda(h \xi))}{|\lambda(h \xi)-\lambda(h \eta)|}\right||\hat{u}(\eta)| d \eta \mid \\
& \leqq h C\left\|\dot{a}_{1}(\xi)|\xi|\right\|_{L_{1}}\|u\|+h\left\|\dot{a}_{1}(\xi)|\xi|\right\|_{L_{1}} S\|u\| \\
& S=\text { Lipshitz constant of }|\xi| k(\xi) \text { for }|\xi| \leqq 1 \text {. }
\end{aligned}
$$

The proof is same for $\left[A, K_{h}\right] \Lambda_{h}$.

Thus we get the following:

Theorem 1 . The set $\Omega$ is a two sided ideal in $\mathscr{P}$.

Prof. For every element $N_{h}$ in $\Re$, we will show that any product $H_{h} N_{h}$ and $N_{k} H_{h}$ belong to $\mathscr{N}$ taking $H_{h}$ in $\mathcal{P}$. Because $H_{h}$ is formed by addition and multiplication from fininite number of families $K_{k}$ associated with $k(x, \xi)$ in $\mathcal{K}$. Then it is sufficient to show that any product $K_{h} N_{h}$ and $N_{h} K_{h}$ belong to $\Re$. On the other hand by the lemma $3,\left[K_{k}, \Lambda_{h}\right] \in \Re_{c}$, therefore we get

$$
\begin{aligned}
& K_{h} N_{h} \Lambda_{h}=K_{h}\left(N_{h} \Lambda_{h}\right) \in \mathscr{I}_{0} \\
& N_{h} K_{h} \Lambda_{h}=\left(N_{h} \Lambda_{h}\right) K_{h}+N_{h}\left[K_{h}, \Lambda_{h}\right] \in \mathcal{I}_{0} .
\end{aligned}
$$

This means $K_{h} N_{h}$ and $N_{h} K_{h}$ are negligible.

c.q.f.d.

Now we state the

Theorem 2. The mapping $\mathscr{H}$ from $\mathcal{K}$ onto $\mathscr{L}$ is a homomorphism with respect to addition and multiplication modulo $\Re$.

Proof. The homomorphism with respect to addition is evident. For multiplication we only need the following: 
Lemma 5. If $k_{1}$ and $k_{2}$ are in $\mathcal{K}$, then $k_{1} \cdot k_{2}=k_{3}$ also belongs to $\mathcal{K}$. If we denote $K_{1 h}, K_{2 h}, K_{1 h} \circ K_{2 h}$ the associated family with $k_{1}, k_{2}, k_{3}$ respectively, then we have

$$
K_{1 h} K_{2 h}-K_{1 h} \circ K_{2 h} \in \mathcal{I} \text {. }
$$

Proof of lemma 5. We can assume as usual that $k_{1}$ and $k_{2}$ are some finite sums of type (7), because of lemma 2 . We put

$$
k_{1}=\sum_{\alpha} a_{\alpha}(x) k_{h \alpha}^{(1)}(\xi), \quad k_{2}=\sum_{\beta} b_{\beta}(x) k_{h \beta}^{(2)}(\xi) .
$$

Then we get

$$
k_{3}=\sum_{\alpha, \beta} a_{\alpha}(x) b_{\beta}(x) k_{r_{\alpha}}^{(1)}(\xi) k_{h \beta}^{(2)}(\xi)
$$

and

$$
\begin{gathered}
K_{1 h}=\sum_{\alpha} A_{\alpha} K_{h \alpha}^{(1)}, \quad K_{2 h}=\sum_{\beta} B_{\beta} K_{h \beta}^{(2)} \\
K_{1 h} \circ K_{2 h}=\sum_{\alpha, \beta} A_{\alpha} B_{\beta} K_{h \alpha}^{(1)} K_{h \beta}^{(2)} \\
K_{1 h} K_{2 h}=\sum_{\alpha, \beta} A_{\alpha} K_{h \alpha}^{(1)} B_{\beta} K_{h \beta}^{(2)} .
\end{gathered}
$$

Therefore we have

$$
K_{1 h} K_{2 h}-K_{1 h} \circ K_{2 h}=\sum_{\alpha, \beta} A_{\alpha}\left(B_{\beta} K_{l \alpha}^{(1)}-K_{h \alpha}^{(1)} B_{\beta}\right) K_{\beta, i}^{(2)} .
$$

If we prove the commutator $\left[B_{\beta}, K_{h \alpha}^{(1)}\right]$ is negligible then the proof of Iemma 5 will finish. But this is the simple consequence of lemma 4. $\mathrm{O}_{2}^{c}$ course, by the proof of lemma 4, we get

$$
\left\|\left[B_{\beta}, K_{h \infty}^{(1)}\right] \Lambda_{h}\right\| \leqq C h
$$

with $C$ determined only by the Lipshitz constant of $\lambda(\xi)$ and $|\xi| k_{\imath}(x, \xi)$ and the $C^{1}$ norm of $k_{i}(x, \xi)$ in $x(i=1,2)$.

Corollary. If $K_{1 h}$ and $K_{2 h}$ are the same families in lemma 5, and $M_{h}$ is $K_{1 h} \circ K_{2 h}$, then $K_{h} \Lambda_{l k}^{2}-K_{1 h} \Lambda_{h} \tilde{K}_{2 i} \Lambda_{k}$ is a null scheme.

Proof.

$$
\begin{aligned}
& M_{h} \Lambda_{h}^{2}-K_{1 k} \Lambda_{h} K_{2 h} \Lambda_{h}=\left(M_{h} \Lambda_{h}-K_{1 h} \Lambda_{h} K_{2 h}\right) \Lambda_{h} \\
= & \left(M_{h}-K_{1 h} K_{2 h}\right) \Lambda_{h}^{2}+K_{1 h}\left[\Lambda_{l k}, K_{2 h}\right] \Lambda_{h} \in \mathcal{I}_{0} .
\end{aligned}
$$

Proposition. The homomorphism in theorem 2 is a*-homomorphism. 
Proof. If $k$ is in $\mathcal{K}$, then $k^{*}$ is also in $\mathcal{K}$, we denote $K_{k}$ and $K_{h}^{\#}$ associated family with $k$ and $k^{*}$ respectively. We can prove $K_{h}^{*}$

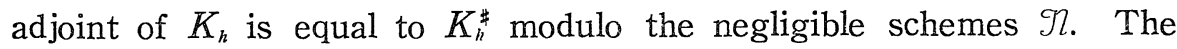
proof of this fact can be proved by the same kind of reasoning as in lemma 5 .

Now we prove the

Theorem 2 '. The homomorphism $\mathcal{M}$ from $\mathcal{K}$ onto $\mathscr{L}$ is an isomorphism modulo $\Re$.

Proof. We are making use of the trick by P. D. Lax [4]. We suppose that there exists a non zero element $k$ in $\mathcal{K}$ whose associated family in $\mathscr{P}$ is negligible. Then for $k^{*} k$, and also for the rotation in $\xi$ of $k^{*}(x, \xi) k(x, \xi)$, the same is true. By the integration with respect to the invariant measure over the whole rotation group. We have a non negative matrix valued function $s(x)$ whose associate family $S_{h}$ is negligible. Therefore $\Lambda_{h} s(x), s(x) \Lambda_{h}$ are null schemes.

On the other hand, because $s(x)$ is a non zero positive semidefinite matrix, we can assume that there exists a component $s_{i i}(x) \geqq \delta>0$ in some open set $\Omega$. If we take a scalar function $u(x)$ which belongs to $\mathscr{D}(\Omega)$, then we get

$$
\left\|\Lambda_{h} s_{i 2}(x) u(x)\right\|=O(h) .
$$

But we can put $s_{i r}(x) u(x)=v(x)$ for any function of $\mathscr{D}(\Omega)$, then we have

$$
\left\|\Lambda_{h} v(x)\right\|=O(h)
$$

which means for some constant $B>0$

$$
\mid \frac{\Lambda_{h} v}{h}\|\leqq B\| v \|, \quad \forall v \in \mathscr{D}(\Omega) .
$$

Consequently by the Lebesgue theorem, we have

$$
\|\Lambda v\| \leqq B\|v\|, \quad \forall v \in \mathscr{D}(\Omega)
$$

that means

$$
\left\|\frac{\partial}{\partial x_{i}} v\right\| \leqq B\|v\|, \quad \forall v \in \mathscr{D}(\Omega)
$$



which is a contradiction.

Now we state a theorem which is important for the later application.

Theorem 3. If $p(x, \xi)$ is positive definite and belongs to $\mathcal{K}$, then the associated family $P_{n}$ satisfies the following inequality for $u \in L_{x}^{2}$.

$$
\mathscr{R} e\left\langle P_{h} \Lambda_{h}^{2} u, u\right\rangle \geqq-O(h)\|u\|^{2} .
$$

Proof. We can find a non singular matrix function $r(x, \xi)$ in $\mathcal{K}$ such that

$$
p(x, \xi)=r^{*}(x, \xi) r(x, \xi) .
$$

Then using corollary of lemma 5 , lemma 2, and lemma 3, we get

$$
\begin{aligned}
\left\langle P_{h} \Lambda_{h}^{2} u, u\right\rangle & =\left\langle\Lambda_{h} R_{h}^{*} R_{h} \Lambda_{h} u, u\right\rangle+O(h)\|u\|^{2} \\
& =\left\langle R_{h} \Lambda_{h} u, R_{h} \Lambda_{h} u\right\rangle+O(h)\|u\|^{2} \\
& \geqq-O(h)\|u\|^{2} .
\end{aligned}
$$

Now we specialize the vector function $\lambda(\xi)$ for the later use. Let us consider a constant coefficient finite difference scheme $D_{j h}$ which approximates the partial derivative $\frac{\partial}{\partial x_{j}}$ with accuracy 1 . Let us write this scheme $D_{j h}=\sum_{l} C_{l} T_{j}^{l}, T_{j} u(x)=u\left(x_{1}, \cdots, x_{j}+h, \cdots x_{n}\right)$. Then we can use $\frac{1}{i} \sum_{l} C_{l} e^{i \xi_{j} l}$, as a $\lambda_{j}(\xi)$ if $\sum_{l} C_{l} e^{i \xi_{j} l}$ is pure imaginary for real $\xi$. In fact by the consistency we can prove

$$
\frac{\lambda_{i}(h \xi)}{h} \rightarrow \xi_{j} \quad \text { for } h \downarrow 0
$$

and $\lambda(\xi)=\frac{1}{i} \sum_{l} C_{l} e^{\xi_{l} l}$ is analytic in $\xi$. Since all derivatives with re spect to $\xi$ are bounded, we have

$$
\left|\lambda_{j}(\xi)-\lambda_{j}(\eta)\right| \leqq C_{j}|\xi-\eta| .
$$

Consequently, we get

$$
|\lambda(\xi)-\lambda(\eta)| \leqq C|\xi-\eta|
$$

where $C$ is a constant independent of $\xi, \eta$ 


\section{Calculus of difference schemes}

Let $S_{k}$ be a family of usual scalar difference operators of the following form:

$$
S_{h}=\sum_{j} b_{j}(x) T^{j} \quad \text { (finite sum) }
$$

where $b_{j}(x)$ is $C^{m}$ and constant for $|x|>R$ and $T^{j}=T_{1}^{j_{1}} \cdot T_{2}^{j_{2}} \ldots \ldots T_{n}^{j_{n}}$, $T_{p_{p}}^{j_{p}} u(x)=u\left(x+j_{p} h e_{p}\right) \quad\left(e_{p}=\left(0 \cdots 01_{p} 0 \cdots 0\right)\right)$. As we have shown in section $2, T_{p}^{j_{p}}$ commute with $a_{j}(x)$ modulo $\Omega_{0}$ and of course $\Lambda_{k}$ commutes with $T_{p}^{j_{p}}$ in usual sense. Thus we have the following.

Lemma 6. Let $K_{h} \Lambda_{h}$ be the family corresponding to $k \in K$, then $K_{h} \Lambda_{h} S_{h}-S_{h} K_{h} \Lambda_{h}$ is a null scheme.

Proof. We use Lemma 1 and Lemma 3 and Lemma 4.

$$
\begin{aligned}
& K_{h} \Lambda_{h} S_{h}=\sum_{j} \sum_{e} a_{j}(x) K_{h j} \Lambda_{h} b_{e}(x) T^{e} \\
& S_{h} K_{h} \Lambda_{h}=\sum_{j} \sum_{e} b_{j}(x) T^{j} a_{e}(x) K_{h e} \Lambda_{h} \\
& a_{j}(x) K_{h j} \Lambda_{h} b_{e}(x) T^{e}-b_{e}(x) T^{e} a_{j}(x) K_{h j} \Lambda_{h} \\
& =a_{j}(x) K_{h j}\left(\Lambda_{h} b_{e}(x)-b_{e} \Lambda_{h}\right) T^{e} \\
& \quad+a_{j}(x)\left(K_{h j} b_{e}(x)-b_{e}(x) K_{h j} \Lambda_{h} T^{e}\right. \\
& \quad+b_{e}(x)\left(a_{j}(x) T^{e}-T^{e} a_{j}(x)\right) K_{h j} \Lambda_{h} \in I_{I_{0}} .
\end{aligned}
$$

We can write:

$$
\begin{aligned}
a_{J}(x) K_{h}, \Lambda_{h} b_{e}(x) T^{e} \equiv & a_{J}(x) K_{h_{j}} b_{e}(x) \Lambda_{h} T^{e} \\
\equiv & a_{j}(x) b_{e}(x) K_{h_{j}} \Lambda_{h} T^{e} \equiv a_{j}(x) b_{e}(x) T^{e} K_{h j} \Lambda_{h} \\
\equiv & b_{e}(x) T^{e} a_{j}(x) K_{h} \Lambda_{h} \quad\left(\bmod \Re_{0}\right) \\
& \text { 5. Application }
\end{aligned}
$$

\section{Application}

The stability of Friedrichs' scheme for regularly lhy perbolic systems.

Let us consider the system of equations with variable coefficients

$$
\frac{\partial u}{\partial t}=\sum_{j=1}^{n} A_{j}(x) \frac{\partial u}{\partial x_{j}}
$$

where $A_{j}(x)$ are given smooth bounded $N \times N$ matrices and equal to 
constant for large $x$ while $u$ is an unknown $N$-vector. We assume that (13) is a regularly hyperbolic system, that is to say, that the matrix $\sum_{j} A_{j}(x) \xi_{j}=A(x, \xi)$ have only real distinct eigenvalues for all real $\xi=\left(\xi_{1}, \cdots, \xi_{n}\right)$ and every pair of eigenvalues $\mu_{p}(x, \xi)$ and $\mu_{q}(x, \xi)$ satisfy the following condition: there exists a positive constant $d$ such that

$$
\begin{array}{ll}
\left|\mu_{p}(x, \xi)-\mu_{q}(x, \xi)\right| \geqq d \quad \text { for all } x \in R^{n}, \xi \in S^{n-1} \\
& (p \neq q) p, q=1, \cdots, N .
\end{array}
$$

Then we know that there is a nonsingular smooth matrix $N(x, \xi)$ whose determinant is bounded away from zero for all $x \in R^{n}, \xi \in S^{n-1}$, and that it is a diagonalizer of $A(x, \xi)$, i.e.

$$
\begin{aligned}
D(X, \xi) & =N(x, \xi) A(x, \xi) N^{-1}(x, \xi) \\
& =\left(\begin{array}{lll}
\mu_{1}(x, \xi) & \\
& \ddots & \\
& & \mu_{N}(x, \xi)
\end{array}\right) .
\end{aligned}
$$

Now we consider the numerical solution of the Cauchy problem for this system. Among useful finite difference schemes, we consider here exclusively one which is called Friedrichs' scheme. Replacing $\frac{\partial u}{\partial r}$ by $u(x, t+k)-\overline{u(x, t)} / k$ and $\frac{\partial u}{\partial x_{j}}$ by $\frac{u\left(x+\delta_{j}, t\right)-u\left(x-\delta_{j}, t\right)}{2 h}$ where $h$ and $k$ are space and time mesh length respectively and we denoted $\overline{u(x, t)}=\frac{\sum_{j=1}^{n}\left\{u\left(x+\delta_{j}, t\right)+u\left(x-\delta_{j}, t\right)\right\}}{2 n}\left(\delta_{j}=h e_{j}, e_{j}\right.$ is a unit $n$-vectors $j=1, \cdots, n)$, we can write down the Friedrichs' scheme for the system (13) as follows: for every $u \in L_{x}^{2}$

$$
\begin{gathered}
u(x, t+k)=\sum_{j=1}^{n}\left[\frac{u\left(x+\delta_{j}, t\right)+u\left(x-\delta_{j}, t\right)}{2 n}\right. \\
\left.+k A_{j}(x) \frac{u\left(x+\delta_{j}, t\right)-u\left(x-\delta_{j}, t\right)}{2 h}\right] .
\end{gathered}
$$

Or denoting $\lambda=\frac{k}{h}$, we have

$$
u(x, t+k)=S_{h} u(x, t) \text {, where } S_{h} \text { is defined by }
$$




$$
\begin{aligned}
S_{h} u & =\sum_{j=1}^{n}\left\{\frac{u\left(x+\delta_{j}, t\right)+u\left(x-\delta_{j}, t\right)}{2 n}\right. \\
& \left.+\lambda A_{j}(x) \frac{u\left(x+\delta_{j}, t\right)-u\left(x-\delta_{j}, t\right)}{2}\right\} .
\end{aligned}
$$

Now we state the stability theorem.

Theorem 3. If $|\lambda|<\frac{1}{\sqrt{n} \mu_{0}}$, then the scheme (17), (18) is stable in the sense of Lax-Richtmyer. Here $\mu_{0}$ is $\underset{\substack{p=1, \cdots, N \\ j \in 1 \\ x \in R^{n}}}{\max }\left|\mu_{p}(x, \xi)\right|$.

Proof of the theorem. First we remark that the stability means for any $m$ positive integer, $\left\|S_{h}^{m} u(x, 0)\right\| \leqq C\|u(x, 0)\|$, where $C$ is a constant independent of $m$ and $h$ and $m h \leqq T$.

To show this inequality it is convenient to introduce a new norm which is equivalent to the usual $L^{2}$ norm. First vie explain its construction and equivalence.

i) Construction of a new norm.

Let $H_{h}$ be a bounded operator corresponding to $h(x, \xi)=$ $N^{*}(x, \xi) N(x, \xi)$ which is a strictly positive definite function for $x \in R^{n}$, $\xi \in S^{n-1}$ and belongs to $\mathcal{K}$. Then we can show for any $u$ with small fixed support, $\left\langle\mathscr{R} e H_{h} u, u\right\rangle$ is positive definite. Because

$$
\begin{aligned}
& \left\langle H_{h} u, u\right\rangle=\left\langle H_{0 h} u, u\right\rangle+\sum_{j=1}^{n}\left\langle\left(x^{j}-x_{0}^{j}\right) H_{1 j h} u, u\right\rangle \\
& H_{0 h}: \text { operator corresponding to } h\left(x_{0}, \xi\right) \\
& H_{1 j h}: \text { operator corresponding to } h_{1 j}(x, \xi) \text { such that } \\
& h(x, \xi)=h\left(x_{0}, \xi\right)+\sum_{j=1}^{n}\left(x^{j}-x_{0}^{j}\right) h_{1 j}(x, \xi) .
\end{aligned}
$$

Naturally $\operatorname{Re}\left\langle H_{0 h} u, u\right\rangle \geqq d_{1}|| u \|^{2}$, then if we take the diameter of that fixed support very small, we can show (although $H_{1 j k}$ doesn't belong to the operator family discussed in §3.)

$$
\left|\left\langle\left(x^{j}-x_{0}^{j}\right) H_{1 j h} u, u\right\rangle\right| \leqq \varepsilon\|u\|^{2} .
$$

We get

$$
\mathcal{R} e\left\langle H_{h} u, u\right\rangle \geqq d_{2}\|u\|^{2}
$$


We fix now a partition of unity $\left\{\varphi_{p}\right\}$ such that $\sum_{p} \varphi_{p}^{2}=1$. Because of the assumption about $A_{j}(x)$, we consider only a finite partition of unity. If we take the maximum of diameter of the support of $\varphi_{p}$ sufficiently small, then

$$
\| u_{H}^{2}=\sum_{j} \mathscr{R} e\left\langle H_{h} \varphi_{j} u, \varphi_{j} u\right\rangle
$$

is equivalent to the $L^{2}$ norm. For the inequality $\|u\|_{H} \leqq c\|u\|$ is evident by the boundedness of $H_{h}$. The inverse inequality results from (20) by summing up the inequality (20) for $\varphi_{j} u$.

Now we can say that it suffices to prove

$$
\left\|S_{h} u\right\|_{H} \leqq(1+O(h))\|u\|_{H}
$$

for the stability of (17). But to establish (22), we only have to show

$$
\mathscr{R} e\left\langle H_{t} S_{k} \varphi_{j} u, S_{i} \varphi_{j} u\right\rangle \leqq(1+O(h)) \mathscr{R} e\left\langle H_{k} \varphi_{j} u, \varphi_{j} u\right\rangle, \quad .
$$

because the left hand side of (22) is

$$
\sum_{j} \operatorname{R} e\left\langle\varphi_{j} H_{h} \varphi_{j} S_{h} u, S_{h} u\right\rangle
$$

and

$$
\begin{aligned}
& \left|\left\langle H_{h} \varphi_{j} S_{h} u, \varphi_{j} S_{h} u\right\rangle-\left\langle H_{h} S_{h} \varphi_{j} u, S_{h} \varphi_{j} u\right\rangle\right| \\
= & \left\langle H_{h}\left(\varphi_{j} S_{h}-S_{h} \varphi_{j}\right) u, \varphi_{j} S_{h} u\right\rangle+\left\langle H_{h} S_{h} \varphi_{j} u,\left(\varphi_{j} S_{h}-S_{h} \varphi_{j}\right) u\right\rangle \mid \\
\leqq & O(h)\|u\|^{2} .
\end{aligned}
$$

ii) Proof of (23). Putting $v=\varphi_{j} u$, we'll show

$$
\mathscr{R} e\left\langle H_{h} S_{l} v, S_{h} v\right\rangle \leqq(1+O(h)) \mathscr{R} e\left\langle H_{h} v, v\right\rangle
$$

that means

$$
\mathcal{R e}\left\langle\left(H_{h}-S_{h}^{*} H_{h} S_{h}\right) v, v\right\rangle \geqq-O(h)\|v\|^{2} .
$$

Now we can write $S_{l}$ and $S_{h}^{*}$ in the following form (here we take $\lambda(\xi)=\sin \xi$ as in the section 2$)$

$$
\begin{aligned}
& S_{h}=E_{h}+i \lambda Q_{h} \Lambda_{h} \\
& S_{h}^{*}=E_{h}^{*}-i \lambda \Lambda_{h} Q_{h}^{*} .
\end{aligned}
$$

Where $E_{l}=\mathscr{F}^{-1}\left[I \cdot \sum_{j=1}^{n} \frac{\cos h \xi_{j}}{n}\right] \mathscr{F}$ and $Q_{h}$ is a family corresponding to 
$\sum^{n} A_{j}(X) \frac{\xi_{j}}{|\xi|} \in \mathcal{K} . \quad$ Now we put $P_{h}=H_{k}-S_{h}^{*} H_{k} S_{b}$ and we get, using (26),

$$
P_{h}=P_{h}^{(0)}+i \lambda P_{h}^{(1)}+\lambda^{2} P_{h}^{(2)}
$$

where

$$
\begin{aligned}
& P_{h}^{(0)}=H_{h}-E_{h}^{*} H_{h} E_{h} \\
& P_{h}^{(1)}=\Lambda_{i} Q_{l}^{*} H_{i} E_{h}-E_{h}^{*} H_{h} Q_{h} \Lambda_{h} \\
& P_{h}^{(2)}=\Lambda_{h} Q_{h}^{*} H_{h} Q_{i} \Lambda_{h} .
\end{aligned}
$$

Then we have $P_{h}^{(1)}=0\left(\bmod \Omega_{0}\right)$. In fact using Lemmas $3,4,5$ and the fact that $Q_{n}^{\ddagger} \circ H_{k}=H_{n} \circ Q_{k}$, we get

$$
\begin{aligned}
\Lambda_{h} Q_{h}^{*} H_{k} E_{l} & \equiv \Lambda_{h} Q_{h}^{*} H_{h} E_{h} \equiv Q_{h}^{*} H_{h} \Lambda_{h} E_{h} \\
& \equiv Q_{h} \circ H_{h} \Lambda_{h} E_{h} \equiv H_{h} \circ Q_{h} \Lambda_{h} E_{h} \\
& \equiv H_{h} Q_{h} \Lambda_{h} E_{h} \equiv H_{h} Q_{h} E_{h} \Lambda_{h} \\
& \equiv H_{h} E_{h} Q_{h} \Lambda_{h} \equiv E_{h} H_{h} Q_{h} \Lambda_{h} .
\end{aligned}
$$

Furthermore by Lemmas 3, 4 and Theorem 1 ,

$$
\begin{aligned}
P_{h}^{(2)} & \equiv Q_{h}^{*} \Lambda_{h} H_{k} Q_{h} \Lambda_{h} \equiv Q_{i}^{*} H_{h} \Lambda_{h} Q_{h} \Lambda_{h} \\
& \equiv Q_{h} \circ H_{h} \Lambda_{h} Q_{h} \Lambda_{h} \equiv Q_{h^{\prime}} \circ H_{h} Q_{h} \Lambda_{h}^{2} \\
& \equiv Q_{h} \circ H_{h} \circ Q_{h} \Lambda_{h}^{2} .
\end{aligned}
$$

And we have also

$$
P_{h}^{(0)} \equiv H_{k}\left(I-E_{i h}^{*} E_{h}\right) .
$$

So that

$$
P_{h} \equiv H_{i}\left(I-E_{k}^{*} E_{i}\right)-Q_{h}^{*} \circ H_{i} \circ Q_{k} \Lambda_{h}^{2} .
$$

The Fourier transform $0 \hat{i} I-E_{k h}^{*} E_{i l}$ is expressed by

$$
I \cdot\left(1-\left(\frac{\sum \cosh \xi_{j}}{n}\right)^{2}\right)
$$

and

$$
\begin{aligned}
& 1-\left(\frac{\sum \cos h \xi_{j}}{n}\right)^{2} \\
= & 1-\frac{\sum \cos ^{2} h \xi_{j}}{n}+\frac{\sum_{j>k}\left(\cos h \xi_{j}-\cos h \xi_{k}\right)^{2}}{n^{2}} \\
= & \frac{1}{n} \mid \sin h \xi^{2}+\frac{1}{n^{2}} \sum_{j>k}\left(\cos h \xi_{j}-\cos h \xi_{k}\right)^{2} .
\end{aligned}
$$


Hence we can write using a notation $T_{j k}=T_{j}+T_{j}^{-1}-T_{k}-T_{k}^{-1}$ and lemma 6:

$$
\begin{aligned}
P_{h} & \equiv \frac{1}{n^{2}} \sum_{j-k} H_{h} T_{j k}^{2}+\left(\frac{1}{n} H_{h}-\lambda^{2} Q_{h}^{\#} \circ H_{i o} H_{h} \circ Q_{h}\right) \Lambda_{h}^{2} \\
& \equiv \frac{1}{n^{2}} \sum_{j>k} T_{j k} H_{h} T_{j k}+\left(\frac{1}{n} H_{h}-\lambda^{2} Q_{h}^{\#} \circ H_{h} \circ Q_{h}\right) \Lambda_{h}^{2},
\end{aligned}
$$

because of the fact

$$
\frac{1}{n} H-Q_{h}^{\#} \circ H_{h} \circ Q_{h}=N_{h}^{\#} \circ\left(\frac{1}{n}-\lambda^{2} \mathscr{D}_{h}^{\#} \circ \mathscr{D}_{h}\right) \circ N_{h}
$$

where $\mathscr{D}_{i}$ is the pseudo difference scheme corresponding to $D(x, \xi)$. Applying the Theorem 3 for the operator $N_{h}^{\#} \circ\left(\frac{1}{n}-\lambda^{2} \mathscr{D}_{n} \circ \mathscr{D}_{h}\right) \circ N_{h}$ whose symbol satisfies the condition of this theorem, we get using the fact $T_{j k}^{*}=T_{j k}$

$$
\begin{aligned}
\operatorname{Re}\left(P_{h} v, v\right)= & \operatorname{Re} \frac{1}{n^{2}} \sum_{j>k}\left(H_{h} T_{j k} v, T_{j k} v\right) \\
& +\operatorname{Re}\left(N_{h} \circ\left(\frac{1}{n}-\lambda^{2} \mathscr{D}_{h}^{*} \circ \mathscr{D}_{h}\right) \circ N_{h} v, v\right) \\
\geqq & \frac{1}{n^{2}} d_{2}\left\|T_{j k} v\right\|^{2}-O(h)\|v\|^{2} \\
\geqq & -O(h)\|v\|^{2} \\
\geqq & -O(h) \mathscr{R e}\left(H_{k} v, v\right) .
\end{aligned}
$$

That means (25), and consequently (22) that was to be proved.

Remark. This method works as well in proving the stability of another scheme. For example, the modified Lax-Wendroff scheme of accuracy 2 which proposed recently by Richtmyer [7], can also be proved to be stable under the same assumption of the coefficients as in (18). This scheme can be written as

$$
S_{l} u=\left\{I+i \lambda Q_{h} \Lambda_{h}\left(E_{h}+i \frac{\lambda}{2} Q_{h} \Lambda_{h}\right)\right\} u .
$$

Essential feature of these schemes is that they are both polynomials of the same matrix $Q_{h}$ that comes from the original system (18). It might be possible to give a general theory for this kind of schemes. 


\section{BIBLIOGRAPHY}

[1] Caldéron, A. P. and A. Zygmund, Singular integral operators and differential equations, Amer. J. Math. 79 (1957), 901-921.

[2] Uniqueness in Cauchy problem, Amer. J. Math. 80 (1958), 16-36.

[3] Lax, P. D., Differential equations, difference equations and matrix theory, Comm. Pure Appl. Math. $\mathbb{1 1}$ (1958), 175-194.

[4] - The $\mathrm{L}_{2}$ operator calculus of Mikhlin, Caldéron and Zygmund, Mimeographed lecture note, New York University.

[5] Yamaguti, M., Sur l'inégalité d'énergie pour le système hyperbolique, Proc. Japan Acad. 35 (1959), 37-41.

[6] Le problème de Cauchy et les opérateurs d'intégrale singulière, Mem. Coll. Sci. Univ. Kyoto Ser. A Math. 32 (1959), 121-151.

[7] Wendroff, B., Well posed problems and stable difference operators, Manuscript. 